\title{
Demografía Y ESTRUCTURA POBLACIONAL DE DIOON ANGUSTIFOLIUM (ZAMIACEAE) EN EL NORESTE DE MÉXICO
}

\section{DEMOGRAPHY AND POPULATION STRUCTURE OF DIOON ANGUSTIFOLIUM (ZAMIACEAE) IN NORTHEASTERN MEXICO}

\author{
Gabriel Rubio-MÉndez ${ }^{1}$, Laura YÁÑez-Espinosa ${ }^{2, *}$, María Magdalena Salinas-RodrígueZ ${ }^{3}$, Karen Abigail \\ HERNÁNDEZ-HERNÁNDEZ ${ }^{1}$ Y DAVID BALDERAS-GONZÁLEZ ${ }^{1}$.
}

\author{
${ }^{1}$ Programas Multidisciplinarios de Posgrado en Ciencias Ambientales, Universidad Autónoma de San Luis Potosí, San Luis Potosí, \\ México. \\ 2 Instituto de Investigación de Zonas Desérticas, Universidad Autónoma de San Luis Potosí, San Luis Potosí, México. \\ ${ }^{3}$ Biología, Facultad de Ciencias Naturales, Universidad Autónoma de Querétaro, Querétaro, México. \\ * Autor para correspondencia: lyaneze@gmail.com
}

\section{Resumen}

Antecedentes: Dioon angustifolium es una especie endémica que se distribuye en el extremo noreste de la Sierra Madre Oriental. Se clasifica como especie en peligro de extinción debido a actividades antrópicas como la extracción ilegal con fines comerciales.

Pregunta: ¿Cuál es el estado actual de la estructura de las poblaciones de Dioon angustifolium en su área de distribución?

Especie en estudio: Dioon angustifolium Miq. (Zamiaceae), especie endémica de la Sierra Madre Oriental en México.

Lugar y años de estudio: El estudio se realizó en tres poblaciones de D. angustifolium en Nuevo León (DA1, DA2) y Tamaulipas (DA3) durante el verano de 2018

Métodos: Se determinó la densidad y estructura poblacional de D. angustifolium mediante transectos de $100 \mathrm{~m}$ de largo. Para cada una de las poblaciones analizadas se construyó una tabla de vida estática.

Resultados: La mayor densidad correspondió a la población DA1 y la menor a DA3. Esta última población está dominada por individuos en fase juvenil. Las tablas de vida indican alta tasa de mortalidad en las primeras etapas del establecimiento, lo que provoca que las poblaciones se encuentren en decremento por la falta de reclutamiento de nuevos individuos.

Conclusiones: La extracción de individuos para su comercialización, así como el cambio de uso de suelo disminuyen el tamaño de las poblaciones. De acuerdo con información proporcionada por lugareños, las semillas son utilizadas para elaborar tortillas y atole, sin que se tengan datos de los volúmenes de extracción para poder cuantificar el efecto sobre la dinámica poblacional.

Palabras clave: Cícadas, demografía, Dioon angustifolium, Sierra Madre Oriental, uso alimentario

\section{Abstract}

Background: Dioon angustifolium is an endemic species distributed in the northernmost Sierra Madre Oriental in Mexico. It is classified as an endangered species due to anthropogenic activities like illegal extraction for commercial purposes.

Question: What is the current status of Dioon angustifolium populations structure in its distribution range?

Species under study: Dioon angustifolium Miq. (Zamiaceae), an endemic species of the Sierra Madre Oriental in Mexico.

Study area and years of study: The study was conducted in three populations of D. angustifolium in Nuevo León and Tamaulipas during the summer of 2018 .

Methods: The density and population structure of $D$. angustifolium was determined by transects of $100 \mathrm{~m}$ long. For each of the analyzed populations, a static life table was built.

Results: The highest density corresponded to the DA1 population and the lowest to DA3. This last population is dominated by individuals in the juvenile phase. The life tables indicate a high mortality rate in the first stages of establishment, which causes the populations to be in decline due to the lack of recruitment of new individuals.

Conclusions: The extraction of individuals for commercialization, as well as the change of land use decreases the size of the populations. According to information provided by locals, seeds are used to cook food like tortillas and atole, without having data on extraction volumes to quantify the effect on population dynamics.

Keywords: Cycads, demography, Dioon angustifolium, food use, Sierra Madre Oriental 
Dioon angustifolium Miq. (Zamiaceae) es la especie de Dioon que se distribuye en el extremo norte de la Sierra Madre Oriental, en los estados de Nuevo León y Tamaulipas. Presenta un alto grado de endemismo debido a que se distribuye en superficies reducidas, principalmente en cañadas y laderas rocosas (López-Ovando 2002, González-Astorga et al. 2005). Se reconoció a $D$. angustifolium como especie con base en la morfología de sus foliolos y la variación genética de 14 loci aloenzimáticos en poblaciones de $D$. edule en su rango de distribución (González-Astorga et al. 2003a, 2003b, 2005). Sin embargo, recientemente se ha rechazado esta aseveración, ya que análisis de la secuencia de las regiones ITS y trnL-F, sitúan a $D$. angustifolium y $D$. edule mezclados en el mismo clado (González et al. 2008). Dioon angustifolium se distribuye en la Sierra Madre Oriental entre los 200 y 1,500 $\mathrm{m}$ snm y presenta la mayor densidad entre los 400 y $850 \mathrm{~m}$ snm. Se encuentra en diferentes tipos de vegetación como bosque de Quercus y matorral submontano, que se refiere aun matorral que se ubica en la parte baja de las montañas y presenta elementos transicionales del matorral espinoso tamaulipeco (Rzedowski 2006).

Al igual que todas las especies de cícadas en el mundo, las poblaciones de Dioon angustifolium se encuentran amenazadas debido a las actividades antrópicas, principalmente el cambio de uso de suelo a suelo forestal y ganadero y la extracción con fines comerciales (López-Ovando 2002), por lo que esta especie se encuentra en la NOM-059-SEMARNAT-2010 como una especie en peligro de extinción (SEMARNAT 2010). El objetivo de este trabajo fue determinar la estructura de tres poblaciones de Dioon angustifolium en dos tipos de vegetación en su rango de distribución. Se describe la demografía y algunas variables alómetricas de esta especie.

\section{Materiales y métodos}

Especie de estudio. Dioon angustifolium es una planta con un tallo de hasta un metro de alto y diámetro de 17.5 a $22.5 \mathrm{~cm}$, las hojas son generalmente rectas y planas de hasta $0.8 \mathrm{~m}$ de largo y $16 \mathrm{~cm}$ de ancho. Los foliolos son rectos y planos de 6 a $11 \mathrm{~cm}$ de largo y de 0.4 a $0.6 \mathrm{~cm}$ de ancho y se encuentran insertados en el raquis en ángulo agudo, imbricados, presentan un color verde oscuro, opaco. Conos masculinos angostamente ovados, pardo-pálidos de $33-40 \mathrm{~cm}$ de largo y 8-10 cm de diámetro. Conos femeninos ovados pardo-pálidos de $40-50 \mathrm{~cm}$ de largo y $20-25 \mathrm{~cm}$ de diámetro. Las semillas son ovadas, de $20 \mathrm{~mm}$ de largo y $20 \mathrm{~mm}$ de diámetro; sarcotesta amarilla o blanca (de Luca et al. 1982, Whitelock 2004, Yáñez-Espinosa 2006)

Área de estudio. El estudio se realizó en tres poblaciones representativas de Dioon angustifolium: en Linares, Nuevo León (poblaciones DA1 y DA2) y Ciudad Victoria, Tamaulipas (población DA3; Figura 1). Las poblaciones de DA1 y DA2 presentan vegetación de matorral submontano con la presencia principalmente de Acacia, Bauhinia, Berberis y Zanthoxylum. En la población DA1 se presenta una pendiente de $29 \%$, litosol con sustrato de caliza-lutita, se encuentra a

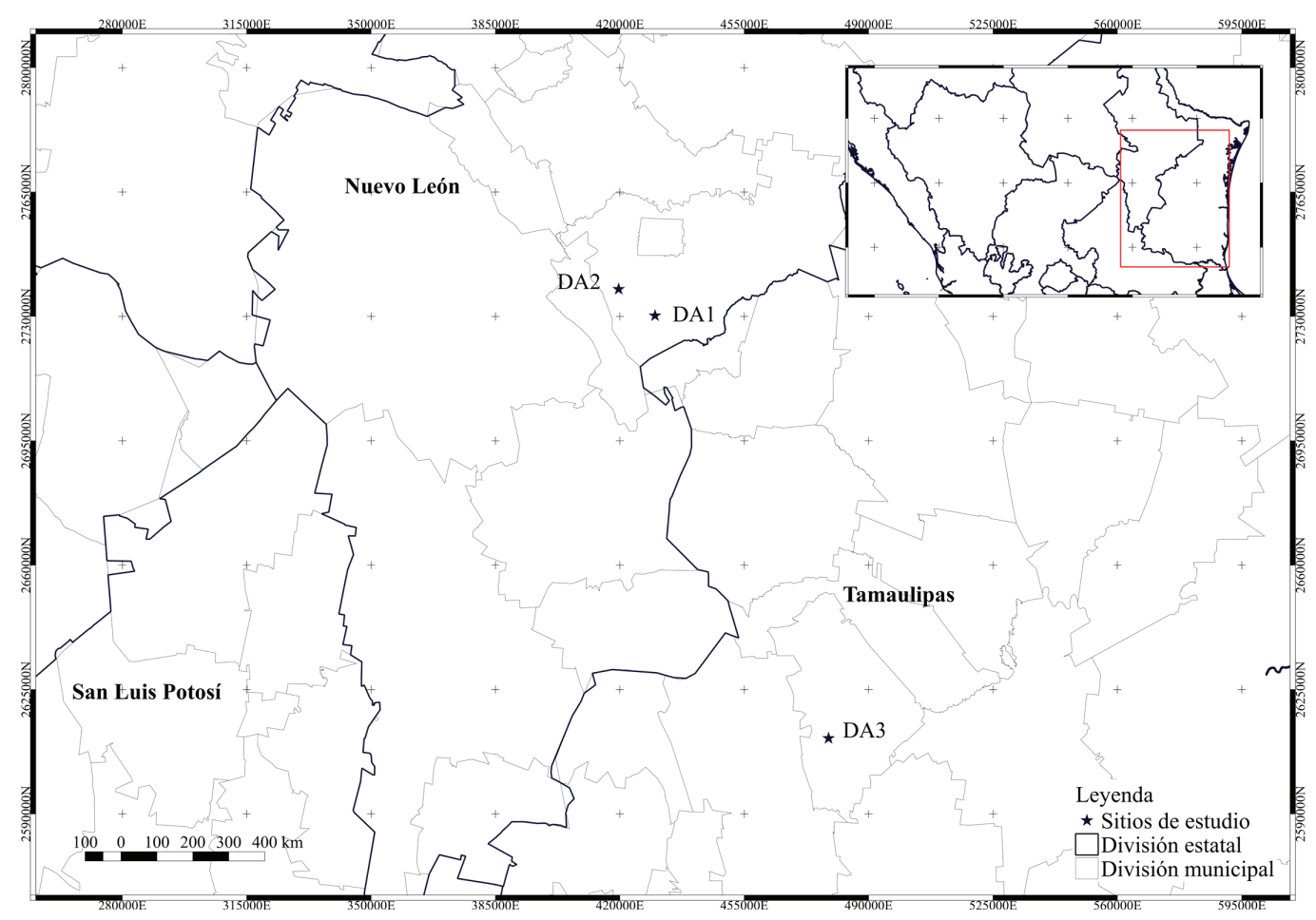

Figura 1. Área de estudio. 
una elevación de 497 m snm, y presenta una precipitación anual de $586 \mathrm{~mm}$, mientras que la población DA2 se encuentra a un costado de un rio intermitente, muy cerca de un núcleo de población y con presencia de actividades antrópicas. En esta población, el suelo es rendzina, con sustrato de lutita, se encuentra a una elevación de $497 \mathrm{~m}$ snm y tiene una precipitación media anual de $754 \mathrm{~mm}$. La población DA3 se encuentra en un bosque de Quercus sp. con pendiente del $14 \%$ y con fase lítica superficial de roca caliza. Se encuentra a una altitud de $925 \mathrm{~m}$ snm y tiene una precipitación media anual de $586 \mathrm{~mm}$; en este sitio se registró la presencia de excretas de bovino (López-Ovando 2002, Salinas-Rodríguez et al. 2013).

Análisis demográfico. Para realizar el análisis demográfico de las poblaciones se trazaron dos transectos de $100 \mathrm{~m}$ de largo y se contaron todos los individuos que interceptaran la línea o se encontraran a una distancia de hasta un metro a cada lado (Chávez-Acuña 2010, Rubio-Méndez 2010). Los individuos registrados fueron clasificados de acuerdo con su estadio de vida como plántulas, juveniles o adultos (Yáñez-Espinosa \& Sosa-Sosa 2007, Rubio-Méndez 2010). Los adultos fueron clasificados como masculino o femenino de acuerdo con la presencia de estructuras reproductoras o como indeterminado en el caso de su ausencia.

Se construyó una tabla de vida estática para cada población tomando las frecuencias de cada estadio de vida y considerando 230 semillas por cono femenino (Mora et al. 2013). Los parámetros demográficos estimados fueron: $l x$, la tasa de sobrevivencia de una edad a la siguiente; $q x$, tasa de mortalidad en el paso de una edad a la siguiente; $e x$, esperanza de vida para cada edad; $R o$, tasa neta reproductiva y $\lambda$, tasa intrínseca de crecimiento poblacional (Valverde et al. 2005, Castillo-Lara et al. 2017, Rubio-Méndez et al. 2018).

Patrones de crecimiento. Para cada individuo registrado se midieron los siguientes parámetros: altura del tallo, diámetro de tallo, número de hojas, número de hojas nuevas (aquellas que se encuentran en la última corona) y longitud de hojas nuevas. Se probaron algunas relaciones alométricas entre estos parámetros para definir patrones de crecimiento por medio de correlaciones simples para probar alguna relación entre variables.

\section{Resultados}

Análisis demográfico. Se registraron un total de 86 individuos en las tres poblaciones, la mayor densidad se encontró en la población DA1 $\left(1,900\right.$ ind $\left.\mathrm{ha}^{-1}\right)$, registrándose también la mayor densidad de plántulas (400 ind ha $\mathrm{h}^{-1}$ ). En la población DA3 se registró la menor densidad de población $(1,000$ ind $\left.\mathrm{ha}^{-1}\right)$. En la población DA2 se encontró el menor número de individuos juveniles (300 ind ha ${ }^{-1}$; Figura 2).

Solo se encontraron ocho adultos reproductivos, dos femeninos DA2, cinco masculinos en DA1 y uno masculino en DA3 (Figura 3). La proporción de sexos para las tres poblaciones fue de 3:1 (m:f).

La mayor tasa sobrevivencia se encontró en la etapa juve-

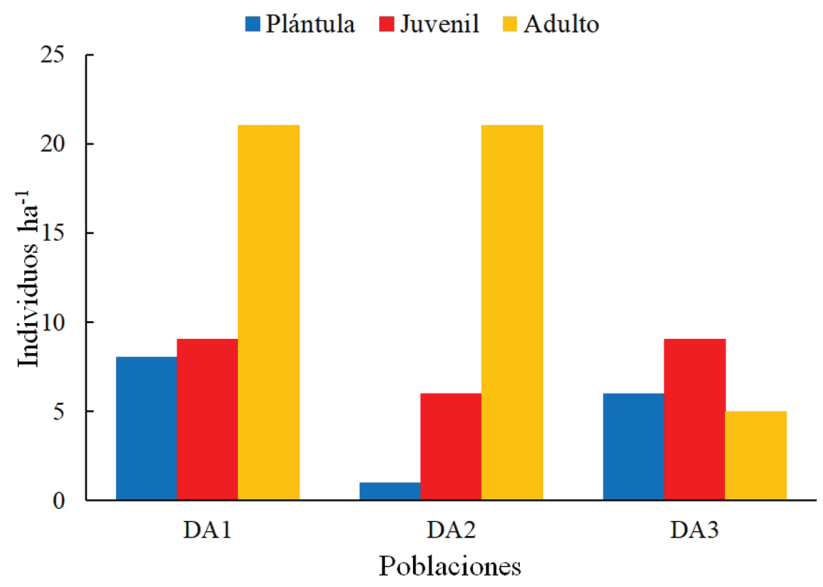

Figura 2. Densidad poblacional de tres poblaciones de Dioon angustifolium.

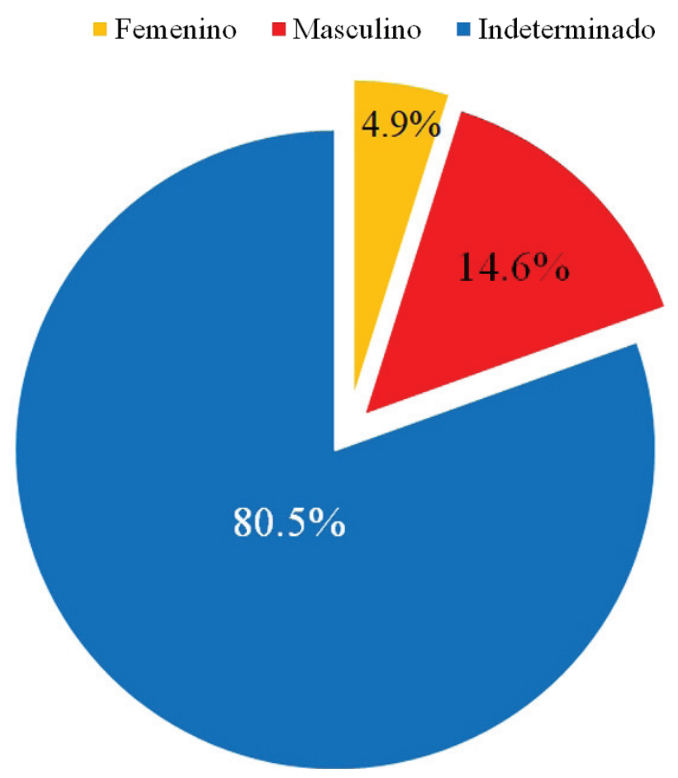

Figura 3. Proporción de sexos en tres poblaciones de Dioon angustifolium.

nil en la población de DA3, seguida por DA1. En general, las mayores tasas de mortalidad se encuentran en la población de DA2 (Figura 4). Aunque en apariencia, las tres poblaciones muestran un crecimiento estable $(\lambda=0.99)$, la tasa neta reproductiva $(R o=0.001)$ y la tasa de crecimiento per cápita es baja $(r<0$; Tabla 1$)$.

Patrones de crecimiento. No se encontraron relaciones alométricas positivas en las poblaciones estudiadas $\left(R^{2}<0.5\right)$. Los individuos más altos, con más hojas y más hojas nuevas se encontraron en DA2, mientras que en DA1 se encontraron los individuos con mayor diámetro y con hojas más grandes (Tabla 2). Los individuos más pequeños y con menos hojas 


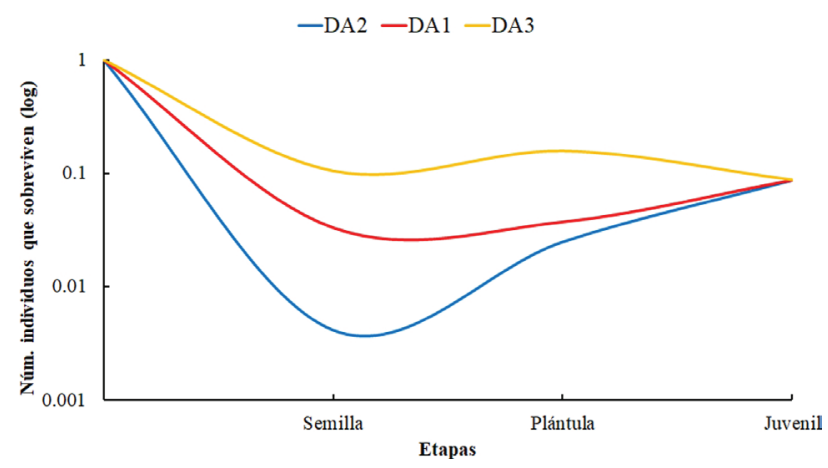

Figura 4. Curva de sobrevivencia para tres poblaciones de Dioon angustifolium.

se encontraron en la población DA3 (Tabla 2), y ésta resultó ser estadísticamente diferente a DA2 en altura $(p=0.025)$.

\section{Discusión}

Las poblaciones de Dioon angustifolium son más pequeñas en densidad en comparación con lo reportado para otras especies del género (Vovides 1990, Negrón-Ortiz et al. 1996, Raimondo \& Donaldson 2003, Pérez-Farrera et al. 2006, Octavio-Aguilar et al. 2008, Cabrera-Toledo 2009). La principal amenaza a la que se enfrentan estas poblaciones son las actividades antrópicas, principalmente al cambio de uso de suelo para agricultura y ganadería. La cercanía a núcleos de población también genera patrones de disturbio caracterizados, entre otras cosas, por la presencia de especies introducidas (Turner et al. 2001), como en el caso de la población DA1, la cual se encuentra muy cerca del núcleo de población $(<200 \mathrm{~m})$ en donde se registró la presencia de la especie Kalanchoe daigremontiana, la cual es utilizada como ornamental.

Los resultados de las tablas de vida muestran que las poblaciones están decreciendo $(r<0)$ como resultado de la falta de reclutamiento de nuevos individuos, lo cual se ve
Tabla 1. Resumen de parámetros demográficos para tres poblaciones de Dioon angustifolium.

\begin{tabular}{clccc}
\hline & & DA1 & DA2 & DA3 \\
\hline \multirow{4}{*}{$l x$} & Plántula & 0.0331 & 0.0041 & 0.1043 \\
& Juvenil & 0.0373 & 0.0248 & 0.1565 \\
& Adulto & 0.0870 & 0.0870 & 0.0870 \\
& Plántula & -0.125 & -5 & -0.5 \\
& Juvenil & -1.333 & -2.5 & 0.444 \\
& Adulto & 1 & 1 & 1 \\
& Plántula & 4.25 & 27.5 & 2.833 \\
& Juvenil & 2.833 & 4 & 1.056 \\
& Adulto & 0.5 & 0.5 & 0.5 \\
& $\boldsymbol{R o}$ & 0.00174 & 0.00174 & 0.00174 \\
& $\boldsymbol{r}$ & -0.00605 & -0.00605 & -0.02542 \\
$\boldsymbol{\lambda}$ & 0.993 & 0.993 & 0.974 \\
\hline
\end{tabular}

reflejado en la baja densidad de plántulas y juveniles. Estas dificultades en el establecimiento pueden estar asociadas a los cambios ambientales que se han producido en las poblaciones (Cabrera-Toledo 2009) como la apertura del dosel, provocada por el cambio de uso de suelo la cual genera una mayor exposición a la radiación solar con un consecuente aumento en la temperatura lo que provoca la deshidratación de las semillas y las plántulas, ya que, en estas etapas, las plantas no cuentan con la suficiente robustez para tolerar estas condiciones (Kitajima \& Fenner 2000, Yáñez-Espinosa et al. 2014, Rubio-Méndez et al. 2018).

La tasa neta reproductiva muestra que las poblaciones analizadas enfrentan problemas reproductivos $(R o<0)$ derivado de la extracción de semillas y/o plantas completas para consumo local o venta. De acuerdo con información proporcionada por habitantes de la región, las semillas de Dioon angustifolium se utilizan localmente en forma de nixtamal para la elaboración de tortilla y atole, sin que exista un registro del volumen de semillas que se extrae. En este

Tabla 2. Parámetros estructurales de tres poblaciones de Dioon angustifolium.

\begin{tabular}{|c|c|c|c|c|c|c|}
\hline Población & & $\begin{array}{l}\text { Altura } \\
(\mathrm{cm})\end{array}$ & $\begin{array}{l}\text { Diámetro } \\
(\mathrm{cm})\end{array}$ & \# Hojas & Hojas nuevas & $\begin{array}{c}\text { Longitud hojas } \\
\text { nuevas (cm) }\end{array}$ \\
\hline \multirow[t]{3}{*}{ DA1 } & Máximo & 55 & 30 & 34 & 15 & 103 \\
\hline & Promedio & 18.71 & 12.95 & 19.19 & 8.61 & 67.03 \\
\hline & E.E. & 3.76 & 2.19 & 1.73 & 0.76 & 598 \\
\hline \multirow[t]{3}{*}{ DA2 } & Máximo & 52 & 34 & 52 & 18 & 98.9 \\
\hline & Promedio & 28.904 & 10.47 & 20.95 & 9 & 66.87 \\
\hline & E.E. & 3 & 2.53 & 2.95 & 0.905 & 5.59 \\
\hline \multirow[t]{3}{*}{ DA3 } & Máximo & 18 & 12 & 26 & 10 & 85 \\
\hline & Promedio & 6.6 & 4.8 & 17 & 5.2 & 56.7 \\
\hline & E.E. & 4.06 & 2.93 & 2.56 & 1.42 & 14.908 \\
\hline
\end{tabular}


sentido es importante documentar el proceso de elaboración de estos productos para poder determinar cuál es el efecto de la extracción de semillas sobre la dinámica de la población.

La tasa intrínseca de crecimiento poblacional indica que las poblaciones están creciendo $(\lambda \approx 0.99)$, sin embargo, esto puede deberse a un efecto de amortiguamiento derivado de la permanencia de los individuos adultos y puede ser resultado de periodos de muestreo cortos que no permiten detectar cambios importantes (Cabrera-Toledo 2009, Caswell 2001).

La tasa de crecimiento de las cícadas varía entre especies dependiendo de varios factores que la afectan, entre los que destacan calidad del suelo, fenología y sexo de la planta (Yáñez-Espinosa 2006). En general, los individuos reproductivos tienen tasas de crecimiento más bajas que aquellos no reproductivos, derivado de un mayor gasto energético durante la reproducción (Clark \& Clark 1988).

Dioon angustifolium presenta una alta plasticidad fenotípica entre sus poblaciones, aunque en general son plantas significativamente más pequeñas que las de $D$. edule, también se encontraron diferencias significativas entre las poblaciones de DA2 y DA3, probablemente como resultado de las diferencias en la calidad del hábitat. Al respecto, Walter \& Schurr (2005) mencionan que los patrones de crecimiento están fijados genéticamente, pero pueden ser modificados por las condiciones ambientales, de tal modo que cambios abruptos en el ambiente afectan directa o indirectamente los patrones de crecimiento.

De acuerdo con nuestros resultados, Dioon angustifolium está enfrentando problemas serios de conservación en su hábitat a pesar de encontrarse catalogada como especie en peligro de extinción de acuerdo con la norma oficial mexicana NOM-059 (SEMARNAT 2010), estos problemas se derivan, principalmente, de la falta de reclutamiento de nuevos individuos. Es necesario realizar estudios a nivel genético para evaluar el nivel de heterocigosis en las poblaciones, flujo genético y depresión por endogamia, derivados de la fragmentación del hábitat.

\section{Literatura citada}

Cabrera-Toledo D. 2009. Biología poblacional de dos cycadas mexicanas con distintos niveles de rareza. $\mathrm{PhD}$. Thesis, Instituto de Ecología, A.C.

Castillo-Lara P, Octavio-Aguilar P, De-Nova J. 2017. Ceratozamia zaragozae Medellín-Leal (Zamiaceae), an endangered Mexican cycad: New information on population structure and spatial distribution. Brittonia 70: 155-165.

DOI: https://doi.org/10.1007/s12228-017-9513-1

Caswell H. 2001. Life Table Response Experiments. In: Caswell H, ed. Matrix Population Models: Construction, Analysis and Interpretation. 2nd edition. Massachusetts: Sinauer, 258278.

Chávez-Acuña IJ. 2010. Análisis estructural de dos poblaciones de Dioon edule Lindl. (Zamiaceae) en comunidades indígenas y mestizas de la región Xi'iuy de La Palma, S.L.P.: Implicaciones ecológicas y culturales. MSc. Thesis. Universidad Autónoma de San Luis Potosí.
Clark DB, Clark DA. 1988. Leaf production and the cost of reproduction in the neotropical rain forest cycad, Zamia skinneri. Journal of Ecology 76: 1153-1163.

DOI: https://doi.org/10.2307/2260640

De Luca P, Sabato S, Vázquez-Torres M. 1982. Distribution and Variation of Dion edule (Zamiaceae). Brittonia, 34: 355-362. DOI: https://doi.org/10.2307/2806710

González-Astorga J, Vovides AP, Iglesias C. 2003a. Morphological and geographical variation of the cycad Dioon edule Lindl. (Zamiaceae): ecological and evolutionary implications. Botanical Journal of the Linnean Society 141: 465-470. DOI: https://doi.org/10.1046/j.1095-8339.2003.00155.x

González-Astorga J, Vovides AP, Ferrer MM, Iglesias C. 2003 b. Population genetics of Dioon edule Lindl. (Zamiaceae, Cycadales): biogeographical and evolutionary implications. Biological Journal of the Linnean Society 80: 457-467. DOI: https://doi.org/10.1046/j.1095-8312.2003.00257.x

González-Astorga J, Vovides AP, Cruz-Angón A, OctavioAguilar P, Iglesias C. 2005. Allozyme variation in three extant populations of the narrowly endemic cycad Dioon angustifolium Miq. (Zamiaceae) from north-eastern Mexico. Annals of Botany 95: 999-1007.

DOI: https://doi.org/10.1093/aob/mci106

González D, Vovides AP, Bárcenas C. 2008. Phylogenetic Relationships of the Neotropical Genus Dioon (Cycadales, Zamiaceae) Based on Nuclear and Chloroplast DNA Sequence Data. Systematic Botany 33: 229-236.

DOI: https://doi.org/10.1600/036364408784571699

Kitajima K, Fenner M. 2000. Ecology of seedling regeneration. In: Fenner M. Ed. Seeds: The ecology of regeneration in plant communities, pp. 331-359. UK: CABI Publishing, Wallingford. ISBN-13: 978-0851994321

López-Ovando A. 2002. Distribución y relaciones ecológicas del chamal (Dioon edule var. angustifolium) en la Sierra de San Carlos, Tamaulipas, Méx. MSc. Thesis. Universidad Autónoma de Nuevo León.

Mora R, Yáñez-Espinosa L, Flores J, Nava-Zárate N. 2013. Strobilus and seed production of Dioon edule (Zamiaceae) in a population with low seedling density in San Luis Potosí, México. Tropical Conservation Science 6: 268-282. DOI: https://doi.org/10.1177/194008291300600208

Negrón-Ortiz V, Gorchov DL, Breckon GJ. 1996. Population structure in Zamia (Zamiaceae) in Northern Puerto Rico. II. Seed germination and stage- structured population projection. International Journal of Plant Science 157: 605-614. DOI: https://doi.org/10.1086/297381

Octavio-Aguilar P, González- Astorga J, Vovides AP. 2008. Population dynamics of the Mexican Cycad Dioon edule Lindl. (Zamiaceae): life history stages and management impact. Biological Journal of the Linnean Society 157: 381-391. DOI: https://doi.org/10.1111/j.1095-8339.2008.00814.X

Pérez-Farrera MA, Vovides AP, Octavio-Aguilar P, GonzálezAstorga J, Cruz-Rodríguez J, Hernández-Jonapá R, Villalobos-Méndez SM. 2006. Demography of the cycad Ceratozamia mirandae (Zamiaceae) under disturbed and undisturbed conditions in a biosphere reserve of Mexico. Plant Ecology 187: $97-108$.

DOI: https://doi.org/10.1007/s11258-006-9135-2 
Raimondo D, Donaldson JS. 2003. Responses of cycads with different life histories to the impact of plant collecting: simulation models to determine important life history stages and population recovery times. Biological Conservation 111: 345358. DOI: https://doi.org/10.1016/S0006-3207(02)00303-8

Rubio-Méndez G. 2010. Estructura Poblacional de Dioon edule en la Sierra Madre Oriental de San Luis Potosí. BSc. Thesis. Universidad Autónoma de San Luis Potosí.

Rubio-Méndez G, Castillo-Gómez HA, Hernández-Sandoval L, Espinosa-Reyes G, De-Nova JA. 2018. Chronic Disturbance Affects the Demography and Population Structure of Beaucarnea inermis, a Threatened Species Endemic to Mexico. Tropical Conservation Science 11.

DOI: https://doi.org/10.1177/1940082918779802

Rzedowski J. 2006. Vegetación de México. México: Comisión Nacional para el Conocimiento y Uso de la Biodiversidad. www.biodiversidad.gob.mx/publicaciones/librosDig/pdf/ VegetacionMx_Cont.pdf (accesed March 11, 2019)

SEMARNAT [Secretaría del Medio Ambiente y Recursos Naturales]. 2010. Norma Oficial Mexicana NOM-059-SEMARNAT-2010, Protección ambiental - Especies nativas de México de flora y fauna silvestres - Categorías de riesgo y especificaciones para su inclusión, exclusión o cambio - Lista de especies en riesgo. Diario Oficial de la Federación. 2da Sección, 30 de diciembre de 2010.

Salinas-Rodríguez MM, Estrada-Castillón E, Villareal-Quintanilla JA. 2013. Flora and Phytogeography of the Cañón De Iturbide, Nuevo Leon, Mexico. Journal of the Botanical Research Institute of Texas 7: 803-819.

Turner MG, Gardner RH, O’Neill RV. 2001. Landscape ecology in theory and practice. New York: Springer-Verlag. ISBN13: 978-0387951225

Valverde T, Cano-Santana Z, Meave J, Carabias J. 2005. Ecología y medio ambiente. Ciudad de México: Pearson Education. ISBN: 9789702605362

Vovides AP. 1990. Spatial distribution, survival, and fecundity of Dioon edule (Zamiaceae) in a tropical deciduous forest in Veracruz, Mexico, with notes on its habitat. American Journal of Botany 77: 1532-1543.

DOI: https://doi.org/10.2307/2444486

Walter A, Schurr U. 2005. Dynamics of leaf and root growth: endogenous control versus environmental impact. Annals of botany 95: 891-900.

DOI: https://doi.org/10.1093/aob/mci103

Whitelock LM. 2004. Variation in the Mexican Cycad Dioon edule (Zamiaceae). The Botanical Review 70: 240-249. DOI:

https://doi.org/10.1663/0006-8101(2004)070[0240:VITMC D]2.0.CO;2

Yáñez-Espinosa L. 2006. Las Cycadas. Biología y conservación en México. Estado de México: Universidad Autónoma de Chapingo. ISBN: 9789680202874

Yáñez-Espinosa L, Sosa-Sosa F. 2007. Population Structure of Dioon purpusii Rose in Oaxaca, México. Neotropical Biology and Conservation 2: 46-54.

Yáñez-Espinosa L, Flores J, Rodríguez-Millán PS, Rubio-Méndez G. 2014. Influence of germination date on Dioon edule (Zamiaceae) seedling tolerance to water stress. Journal of Plant Research 127:413-422.

DOI: https://doi.org/10.1007/s10265-014-0624-4

\section{Editor de sección: Enrique Jurado}

Contribuciones de los autores: GRM (https://orcid.org/0000-00015674-7443) diseñó el muestreo, tomó datos en campo, analizó los datos, escribió el manuscrito. LYE (https://orcid.org/0000-0001-52113603) diseñó el muestreo, tomó datos en campo, analizó los datos, revisó el manuscrito. MMSR (https://orcid.org/0000-0001-7552-7809) guía en campo, tomó datos en campo, revisó el manuscrito. KAHH tomó datos en campo, depuró base de datos, revisó manuscrito. DBG tomó datos en campo, revisó el manuscrito. 